\title{
The Photomotor Response - Dynamic Quantification by a Portable Pupillometer
}

\author{
Jurij Rosen ${ }^{1,2}$, Claudio Privitera ${ }^{3}$, Resul Bulmus ${ }^{1}$, Makoto Nakamura ${ }^{1}$, Alexander Hartmann ${ }^{1,}$ * \\ ${ }^{1}$ Department of Neurosurgery Cologne Merheim, University of Witten/Herdecke, Cologne, Germany \\ ${ }^{2}$ Department of Neurology, Faculty of Medicine and University Hospital Cologne, University of Cologne, Cologne, Germany \\ ${ }^{3}$ School of Optometry, University of California, Minor Hall, Berkeley, the United States
}

Email address:

hartmanna@kliniken-koeln.de (A. Hartmann)

${ }^{*}$ Corresponding author

\section{To cite this article:}

Jurij Rosen, Claudio Privitera, Resul Bulmus, Makoto Nakamura, Alexander Hartmann. The Photomotor Response - Dynamic Quantification by a Portable Pupillometer. American Journal of Internal Medicine. Vol. 8, No. 5, 2020, pp. 230-236. doi: 10.11648/j.ajim.20200805.17

Received: July 17, 2020; Accepted: August 12, 2020; Published: August 31, 2020

\begin{abstract}
The pupillary light reflex (PLR) is a key component of the physical examination as it reliably tests the functional integrity of the neuromuscular loop between pupil and midbrain. Unlike the traditional manual testing the PLR with a penlight that frequently leads to incorrect interpretation due to its subjectivity, the specialized "pupillometer" tool allows objective testing and trending of pupillary data. We performed quantitative unilateral pupillometry several times in 53 healthy subjects (aged 21-74) in different background illumination levels using the NeurOptics NPi®-200 pupillometer. A number of key parameters describing the PLR were collected and analysed. We found that the individual PLR was very consistent. In general, constriction velocity (the first part of the PLR when the pupil constricts promptly after the onset of a light stimulus) was brisker than the dilation velocity (the second part of the PLR, when the pupil recovers from the constriction). Most importantly, both velocities depend on the initial pupillary resting size. We proved that pupillary parameters depend on environmental light conditions and age, but not gender, and scrutinized the nature and dynamics of anisocoric pupils. Taking together, pupillometry is becoming an important, non-invasive clinical tool for testing the autonomic nervous system. Here, we describe baseline parameters representing the physiological PLR, confirming and extending previously reported data. We thus provide the clinician important criteria to precisely assess the PLR and hence the autonomic nervous system in different pathological conditions such as diabetes, traumatic brain injury or cardiac and other autonomic neuropathies.
\end{abstract}

Keywords: Quantitative Pupillometry, Pupillary Light Reflex, Pupil Size, Pupillary Dynamics, Neurological Pupil Index, Diabetes, Cardiac Neuropathy

\section{Introduction}

The pupillary light reflex (PLR) is routinely tested in the neurological examination as it allows painless non-invasive assessment of the integrity of the optic and oculomotor nerve and its presence confirms a functional uninterrupted neuronal pathway through the pretectum and upper midbrain [1]. Despite its importance, the PLR is traditionally been examined by using a penlight, an approach that is prone to both subjectivity and measuring errors and does not allow detailed analysis or trending of the pupillary dynamics [2-5]. Infrared pupillometry is a technology for objectively testing, analysing and understanding the dynamics of the PLR that has become accessible to clinicians in both physiological and pathological conditions $[1,2]$. The technology is based on infrared video recording of the pupillary photomotor response induced by a standardized retinal light stimulation. The entire recording is completed in a few seconds and several pupil parameters, representing the dynamic characteristics of the PLR, are calculated and reported for each measurement (Table 1). Such a standardized procedure allows a detailed analysis of the PLR and its trending over repeated sequential measurements.

Normative values for some of these pupil parameters, such as pupillary resting size or size after light-induced constriction, the corresponding amplitude and speed of the constriction and the subsequent dilation have already been 
published by others $[5,6]$. Other aspects, such as the interdependence between these parameters or their dependence on age have only partly been described, sometimes with contradictory results [1, 5-8]. It is a known fact that pupil size and its dynamics upon retinal light stimulation depend on multiple factors, such as light intensity, age, neurological status, medications or intracranial processes $[1,6]$. However, these factors cannot explain the divergence found in some of the published data.

Other important aspects, such as the consistency of the PLR over time, its correlation with gender and the frequency and extent of physiological anisocoria still need to be investigated. Pupillometry represents a tool to quickly and non-invasively test the activity of the autonomic nervous system and it is becoming a standard procedure in many clinical applications. Thus, normative values for the PLR need to be put forward for interpreting the PLR in different clinical conditions. The objective of this report is to provide normative, quantitative references through an investigation of the physiological PLR in healthy individuals.

\section{Methods}

\subsection{Pupillometer}

The hand-held, cordless NPi®-200 (NeurOptics Inc., Irvine, CA, USA) was used for our quantitative unilateral
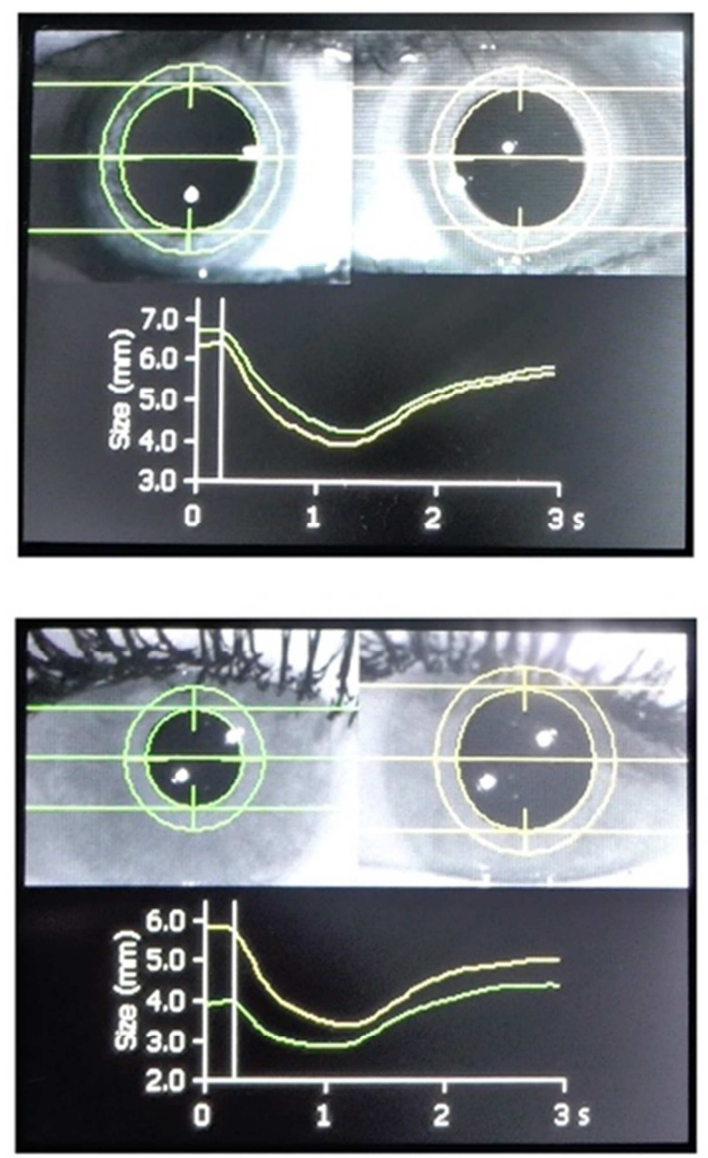
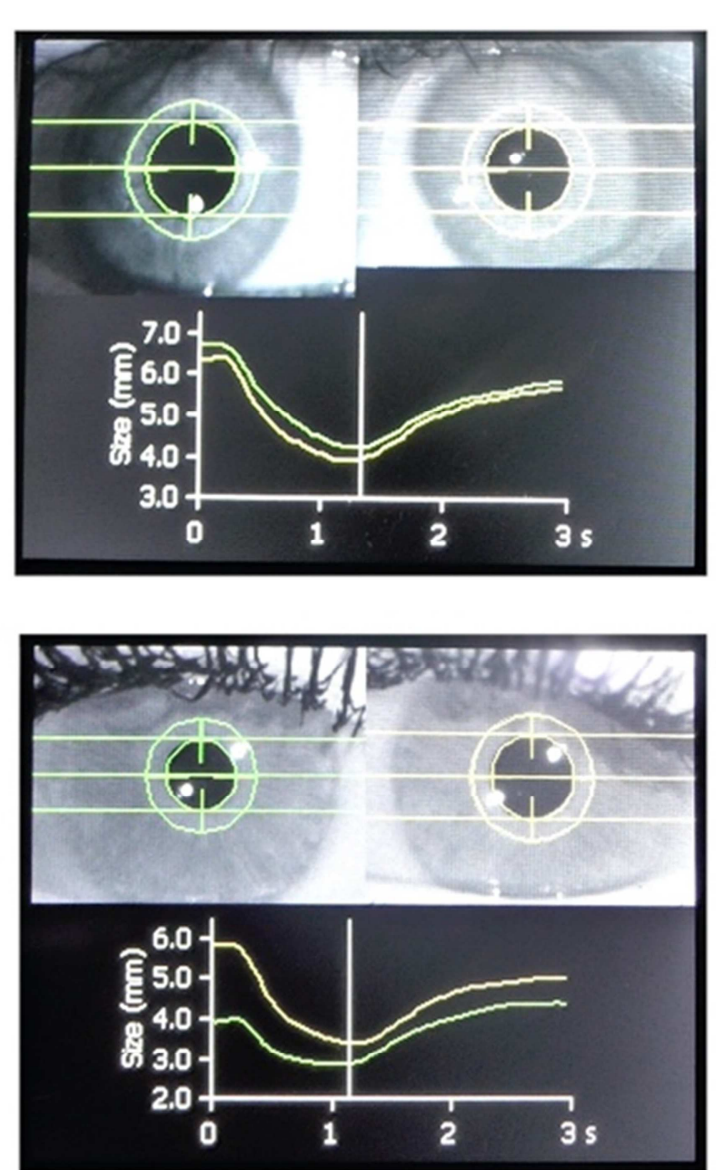

pupillometry. The pupillometer operates at 30 frames per second, pupil tracking is based on standard long infraredlight technology and the measurement, lasting $3 \mathrm{~s}$, is entirely automatized. A pulse of white, LED-emitted light is used to stimulate the pupil reflex; its intensity (approximately 1,000 LUX) and duration (0.8 s) was determined after proper simulation and normative studies [9] to optimize the balance between pupil response sensitivity and perceptive discomfort in all ambient conditions. Its spectral distribution, $400 \mathrm{~nm}$ to $750 \mathrm{~nm}$, is typical in the majority of commercially available white LEDs. Whereas unilateral retinal light stimulation induced bilateral pupillary reaction, only the direct light reaction of the ipsilateral pupil was detected, analysed and recorded (Figure 1). Using this testing model, 7 parameters describing the pupillary light reflex (PLR) are immediately reported by the pupillometer (Table 1). Among these parameters, the Neurological Pupil index (NPi) is automatically calculated. This index was developed to quantify the reactivity of the PLR and thus remove subjectivity that is typical of all manual pupil assessments. All the dynamic variables of the pupil reflex, latency, acceleration, velocity, amplitude, are included in the NPI index and automatically compared to a normative model. The 0-5 scale takes into account this comparison and the overall waveform of the pupil light reflex. More details can be found in $[2,10]$.

Figure 1. Infrared snapshots of recorded pupils during pupillometry. 
Legend to figure 1 The unilateral pupillary light reflex (PLR) was analysed using the hand-held pupillometer NPi $\AA-200$. The pupil was tracked and recorded for three seconds after an ipsilateral $0.8 \mathrm{~s}$ long white light impulse induced the PLR. The same measurement was repeated for the contralateral pupil. Finally, the pair of the left and right pupillary recordings was displayed. Snapshots show two test persons with isocor pupils (upper panel) and with anisocor pupils (lower panel) at rest (left) and after light induced constriction (right, the vertical white bar in the plots indicates elapsed time). The extent of anisocoria (5.8 $\mathrm{mm}$ vs. $4 \mathrm{~mm}$ at rest, bottom left) of a healthy female test person was reduced but still present upon constriction (3.4 mm vs. 2.9 $\mathrm{mm}$, bottom right right). Besides size differences, the PLR (bottom) is within the physiological range (both Neurological Pupil indices normal, 4.4 vs. 4.2 ) confirming the healthy nature of the anisocoria.

Table 1. Pupillary indices and calculated values.

\begin{tabular}{ll}
\hline Index / Value & Description \\
\hline Pupil resting size & Maximal pupillary diameter before light induced pupillary constriction (mm) \\
Size after constriction & Minimal pupillary diameter after light induced pupillary constriction (mm) \\
Constriction velocity & Mean of pupillary constriction velocity (mm/s) \\
Maximal constriction velocity & The maximum velocity recorded during the constriction (mm/s) \\
Dilation velocity & Mean of pupillary dilation velocity (mm/s) \\
Latency & Time interval between light pulse and onset of pupillary constriction (s) \\
Neurological Pupil index (NPi) & Scalar value (0 to 5) summarizing extend and time course of the PLR \\
Absolute constriction amplitude (ACA) & Pupil resting size-size after constriction (mm) \\
Relative constriction amplitude (RCA) & (Pupil resting size-size after constriction)/Pupil resting size (\%) \\
\hline
\end{tabular}

Legend to table 1 By testing the direct pupillary light reflex (PLR) with the pupillometer NPi ${ }^{\circledR}-200$, the first seven indices were reported describing the extend and time course of the PLR. The below listed values (ACA and RCA) were subsequently calculated for further analysis.

\subsection{Pupillary Examination}

All subjects gave informed consent. The study and data accumulation were carried out with prospective approval from the Institutional Review Board (Ethical Committee of the Hospitals of the City Cologne). The study was in accordance with the declaration of Helsinki. In the main experiment testing took place in a constant semi-dark environment (light intensity of 10 lux) after the test person had sufficient time for dark adaption of the eyes. In order to examine the PLR in bright environmental conditions, an external light source was directed at the contralateral pupil (30 lux) and pupillometry subsequently started. During testing, the test person was asked to look straight ahead and to fix a distant object. Both eyes were sequentially tested and testing was repeated for each eye. This testing model was repeated for several days for the same test person. Testing was done in 53 healthy test persons aged 21-74 (31.15) with no significant medical history including ophthalmological disorders or medication affecting pupillary dynamics. Besides testing the PLR, heart rate was measured to assess the test person's sympathetic activity. Measurements of test persons having a heart rate $>100 /$ min were excluded to assure that changes in pupillary dynamics were not caused by high sympathetic activity.

\subsection{Data Processing and Statistical Analysis}

Results are shown as mean $\pm \mathrm{SD}$ and do reflect averaged values from both eyes after repetitive testing. Paired t test was calculated to test for significant differences using $R$ software [11].

\section{Results}

We first analysed the consistency of pupillary indices of the individual test persons. Therefore, differences between the left and right eye and variability between sequential measurements (on the same day and on different days) were calculated for each test person. We could show that data of pupillary dynamics of one individual test person did not exhibit a variation exceeding $10 \%$ between both eyes or over time. As thus the pupillary light reflex (PLR) was proved to be consistent over time and symmetric, indices of the two eyes measured at different time points were averaged together for each test person.

As expected, retinal light stimulation induced pupillary size reduction. On average, pupils constricted from initially $5.4 \pm 1 \mathrm{~mm}$ to $3.3 \pm 0.6 \mathrm{~mm}$ corresponding to a relative constriction amplitude (RCA) of $38.9 \pm 4.2 \%$. The corresponding absolute constriction amplitude (ACA, i. e. the difference between pupil resting size and size after constriction) showed consistent correlation with the pupil resting size, in a way that pupil sizes of different test persons all constricted approximately to the same size after retinal light stimulation. Time latency between the onset of the retinal light stimulation and the onset of pupillary constriction averaged to $0.23 \pm 0.02 \mathrm{~s}$ and did not show any correlation to pupil resting size. Comparing pupil sizes between genders, women exhibited slightly (but not significantly) bigger pupils at rest ( 5.5 vs. $5.2 \mathrm{~mm}, \mathrm{p}=0.08$, paired t-test), a difference that roughly persisted after light induced constriction ( 3.4 vs. $3.2 \mathrm{~mm}, \mathrm{p}=0.03$ ). Almost no difference in RCA was observed $(38.6 \%$ vs. $39.3 \%$ for women and men respectively).

Pupils exhibited heterogeneous dynamics during the different phases of the PLR. During constriction, there is an 
initial strong acceleration followed by a slowdown when the pupil approaches its minimal diameter. Average velocity is simply ACA divided by the time taken by the pupil to cover the entire extension of the constriction. The maximum velocity recorded during the constriction is also reported as a separated index. On average, velocity during constriction was higher than during dilation $(3.1 \pm 0.5$ vs. $1.3 \pm 0.2 \mathrm{~mm} / \mathrm{s}, \mathrm{p}<$ 0.001 , Figure 2 left). Velocities correlated with ACA and pupil resting size in a phase dependent manner. In fact, velocities describing the constriction showed higher correlation with the ACA and pupil resting size than the dilation velocity (Figure 2 middle and right). We found no difference in comparing velocities between genders. Note that dilation is always more problematic than constriction to analyse because sporadic blinking often incurred at the end of the analysed light reflex.

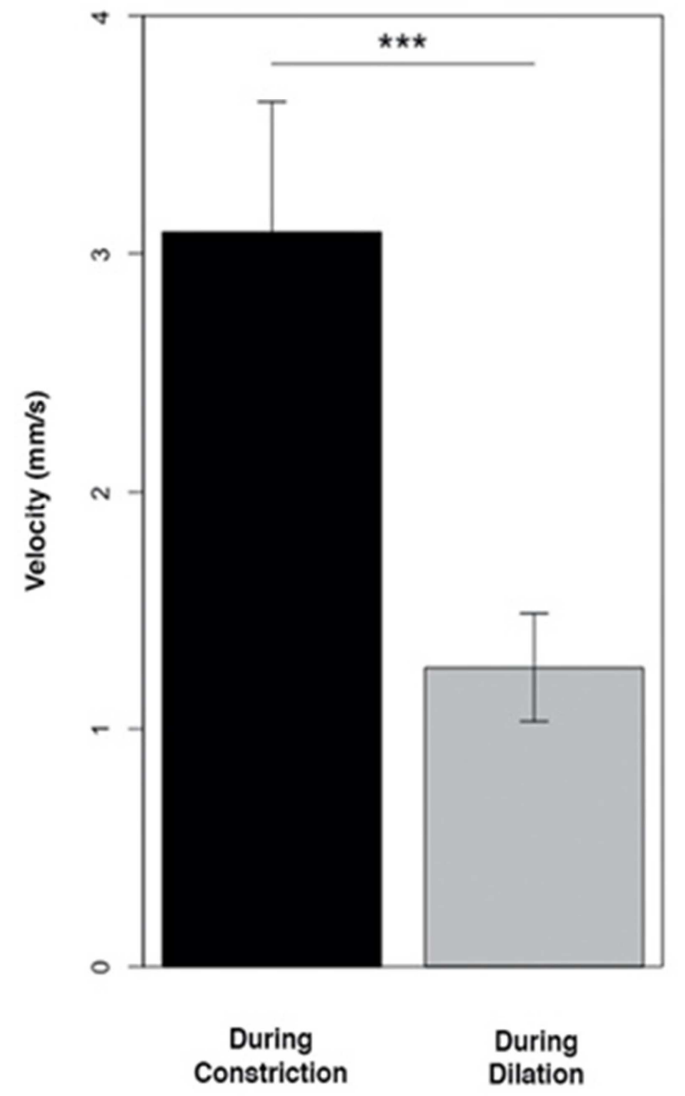

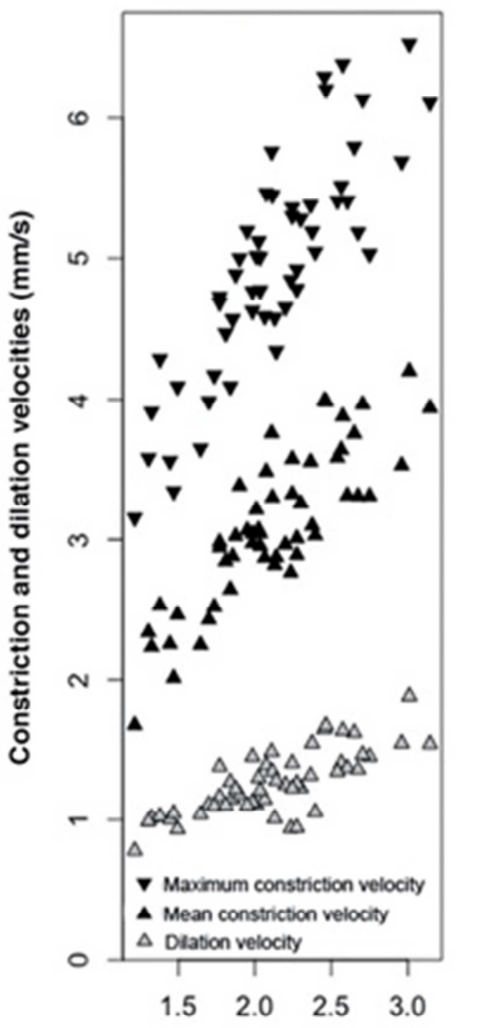

Absolute constriction amplitude ( $\mathrm{mm})$

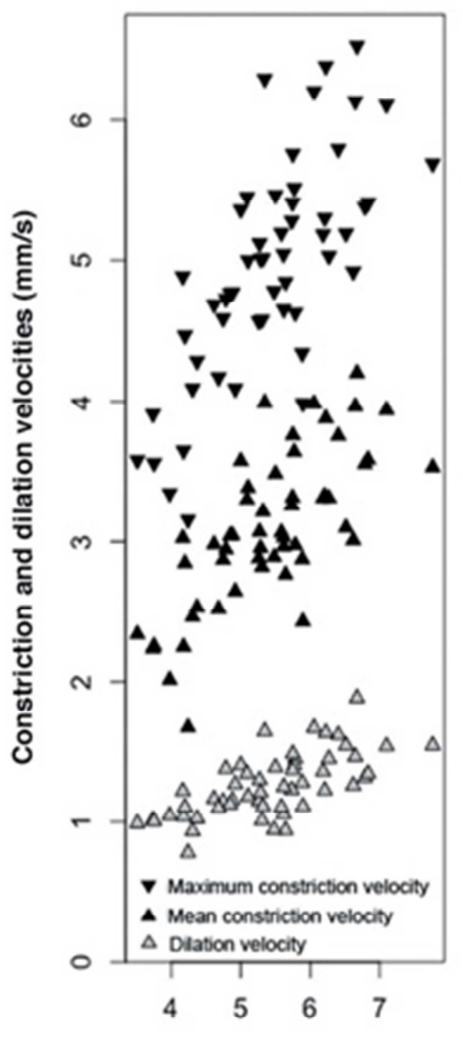

Pupil resting size $(\mathrm{mm})$

Figure 2. Constriction and dilation velocities.

Legend to figure 2 Left panel: Pupillary constriction is on average 2.5 times faster than subsequent dilation $(p<0.001$ paired t-test, bar plots show mean $\pm \mathrm{SD}$ of all measurements). Middle and right panel: Each triangle refers to the averaged maximum/mean constriction velocity (black triangles pointing down/up) or dilation velocity (gray triangle pointing up) of a test person. The individual constriction and dilation velocities show a significant correlation with both the absolute constriction amplitude and pupil resting size.

The calculated Neurological Pupil index (NPi) was on average $4.3 \pm 0.3$, supporting the manufacturer's indication that an NPi $>3$ is supposed to reflect a normal pupillary reactivity (Figure 3). In our population, the NPi ranged from a minimum of 3.3 to 4.8 and it was stable over subsequent measurements, never diverging more than $3 \%$ in the individual test person. Moreover, our data confirmed the age dependency of pupillary size and dynamics. Pupillary resting size, size after constriction and both velocities all decreased with increasing age (Figure 4). Latency, however, showed no dependence on age.

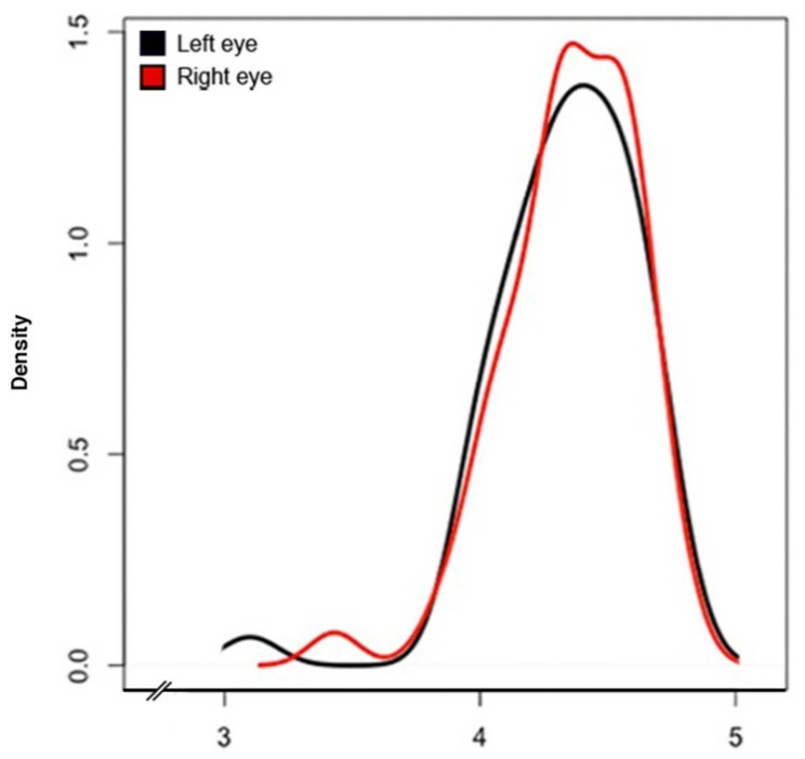

Figure 3. Neurological Pupil index (NPi). 
Legend to figure 3 The NPi index is based on the plurality of all the pupillary indices; calculated for the left and right eye its average is 4.34 and $4.35 \quad( \pm 0.3, \quad$ mean \pm SD $)$

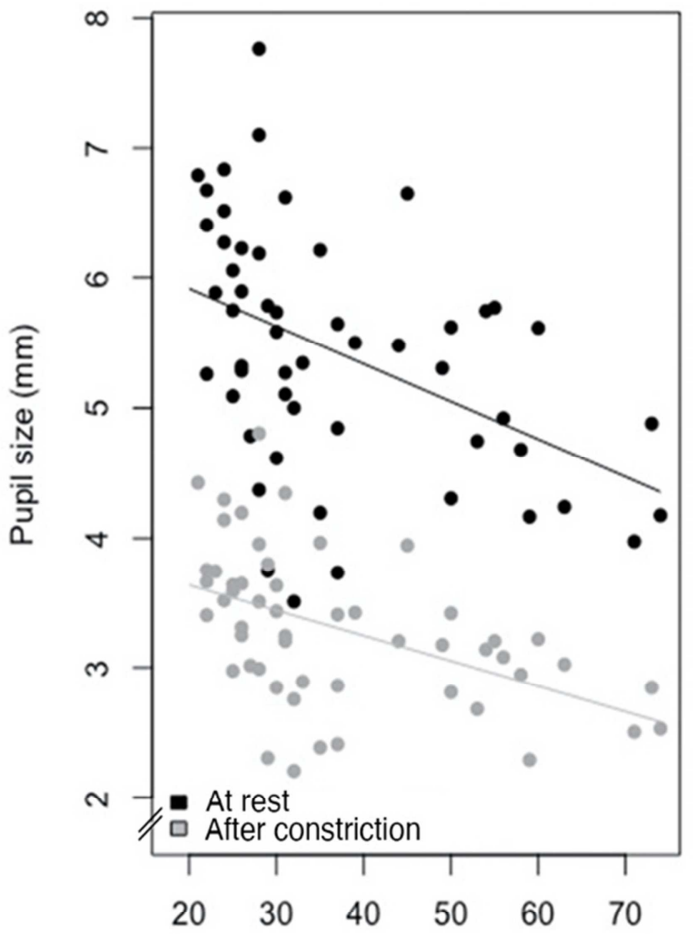

Age respectively. None of the averaged measurements in our healthy population resulted in an $\mathrm{NPi}<3$.

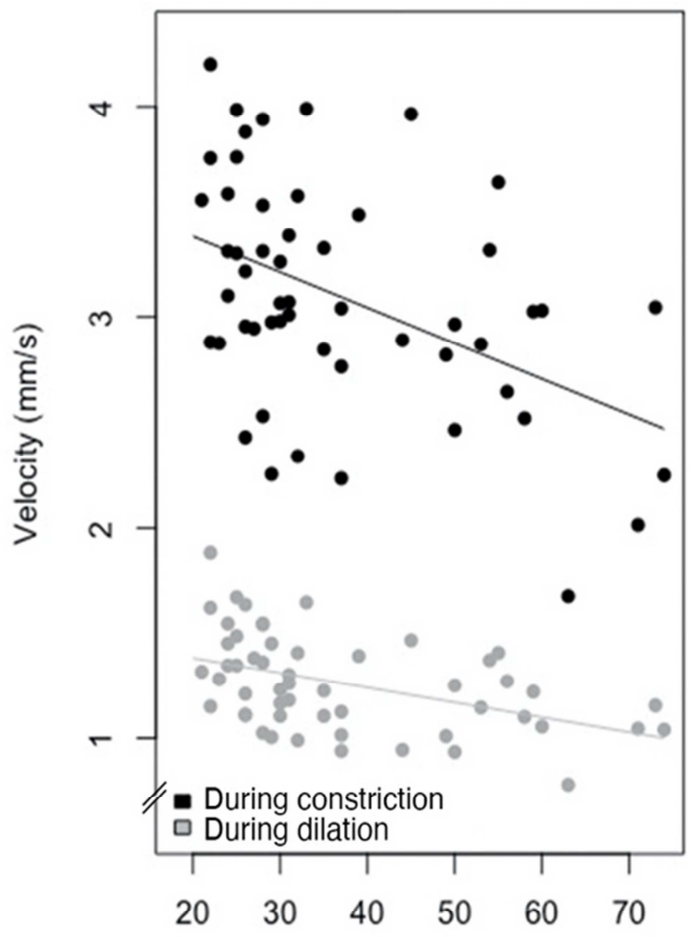

Age

Figure 4. Age dependency of pupil dynamics.

Legend to figure 4 Each point refers to the averaged pupillary parameter of an individual test person with a given age. Left panel: Every 10 years of age, pupil size decreases by an average of $0.3 \mathrm{~mm}$ at rest and $0.2 \mathrm{~mm}$ after light induced constriction. Right panel: Velocity of light induced pupillary constriction and subsequent dilatation also showed age dependency. Every 10 years of increasing age, decreases mean velocity of pupillary constriction and dilation by 0.2 and $0.1 \mathrm{~mm} / \mathrm{s}$ respectively. Values represent averaged bilateral pupillary parameters of $\mathrm{N}=53$ healthy subjects who were tested twice on several days in constant dark light conditions.

Anisocoria, defined as a stable unilateral pupil size difference greater than $0.4 \mathrm{~mm}$, was found in 13 of $53(25 \%)$ test persons at rest with no preference for gender $(22 \%$ of male and $26 \%$ of female) or age ( $34 \mathrm{y} \pm 15)$. Within this group, mean anisocoria in the dark was $0.58 \pm 0.17 \mathrm{~mm}$. Upon constriction, the sign and presence of anisocoria persisted but its extent roughly halved to $0.27 \pm 0.14 \mathrm{~mm}$.

Pupillometry revealed some dependencies for different light conditions (Table 2). As expected, additional exposure to light during pupillometry in a bright environment reduced both pupillary resting size and size after constriction. RCA in direct light exposure was also reduced; same observation for both constriction and dilation velocities and finally for NPi. Latency of pupillary reaction, however, was independent of external light intensity, confirming its lack of correlation with the pupil resting size.

Table 2. Influence of environmental light conditions on pupillary dynamics.

\begin{tabular}{|c|c|c|c|}
\hline \multirow{2}{*}{ Pupillary index } & \multicolumn{2}{|c|}{ Light environment } & \multirow{2}{*}{$P$-value } \\
\hline & Dark & Bright & \\
\hline Pupil resting size (mm) & $6.0 \pm 0.9$ & $3.1 \pm 0.6$ & $\mathrm{p}<0.001$ \\
\hline Size after constriction (mm) & $3.5 \pm 0.6$ & $2.5 \pm 0.4$ & $\mathrm{p}<0.001$ \\
\hline Dilation velocity $(\mathrm{mm} / \mathrm{s})$ & $1.3 \pm 0.3$ & $0.7 \pm 0.3$ & $\mathrm{p}<0.001$ \\
\hline Latency (s) & $0.23 \pm 0.02$ & $0.23 \pm 0.03$ & ns \\
\hline Neurological Pupil index (NPi) & $4.5 \pm 0.2$ & $4.1 \pm 0.4$ & $\mathrm{p}<0.001$ \\
\hline
\end{tabular}

Legend to table 2 Quantitative pupillometry revealed differences in pupillary dynamics in different environmental light conditions. Compared to dark environmental conditions
(Dark), in direct external light exposure (Bright), additional retinal light stimulation by the pupillometer still caused pupil constriction, yet with decreased absolute/relative constriction 
amplitudes. Meanwhile, latency of pupil reaction was unchanged. Velocities of the induced light reflex were roughly halved in direct light exposure. Correspondingly, the NPi also decreased, but only to a small extent and thus with no effect on its clinical interpretation.

The displayed values (mean $\pm \mathrm{SD}$ ) were calculated by testing a subpopulation of $\mathrm{N}=18$ healthy test persons first in the dark and subsequently in direct light exposure (Bright). The level of significance was tested by the paired t-test.

\section{Conclusions}

The objective of the present study was to investigate the dynamics of the pupil light reflex in healthy individuals (physiological PLR). We analysed a collection of pupillary indices that characterizes the motor response of the pupil to a defined light stimulus and evaluated their interdependency and consistency over consecutive measurements. We found these indices to be very consistent. In fact, they never differed more than $10 \%$ in the same light conditions and for sequential measurements in all our subjects. Note that such residual variation is expected and due to several possible physiological causes like the "hippus", a phenomenon referring to natural pupil size oscillations with amplitudes of less than $0.5 \mathrm{~mm}$ [12]. Our data thus confirm consistency over repetitive measurements of the PLR $[1,5,12]$.

Comparing our data with earlier studies, we found both correspondences and discrepancies [5, 7, 8, 13-15]. For example, our range of pupil resting size is in line with Muppidi et al. 2013 [6] and Rickmann et al. 2017 [15], but $25 \%$ larger than data reported by Taylor et al. 2003 [5]. Regarding the RCA, other authors [3] described a wide range of RCA values depending on the pupil resting size, a fact that cannot be reflected by our data. Our results for maximum constriction velocity confirm those published by Bremner et al. 2012 [7] and Muppidi et al. 2013 [6], however, for mean constriction velocity, our results differ by about $50 \%$ from Taylor et al. 2003 [5], probably because of a different distribution of pupil resting sizes in their population likely caused by differences of light conditions or medications.

Pupillary constriction was consistently faster than the subsequent dilation. Physiologically, constriction needs to be brisk to prevent high luminance to damage the retina, whereas effective dilation assures that retinal photoreceptors are optimally exposed to the external light in a darker environment. As our data suggest, preventing damage to the retina was a more impellent evolutionary goal. This thesis is confirmed by the relative anatomic weakness of the radial iris dilator muscle compared to the circular sphincter muscle [1, 16]. Our data confirmed that the constriction and dilation velocities depend on both ACA and pupil resting size as other authors also indicated $[1,5,7,13,14]$. This points to another physiological characteristic of the PLR: The bigger the pupil resting size, the more (ACA) and the faster (constriction velocity) the pupil needs to constrict after light stimulation to effectively reach a smaller diameter to eventually prevent retinal damage.
We confirmed that the dynamics of the PLR depend (as other factors in the autonomic nervous and muscular system) on age $[1,5,6,8,15,17,18]$. Taken together, our data showed that the younger the person, the darker the environment, the bigger the pupil resting size and the correspondent ACA, the faster the constriction and subsequent dilation velocity. Latency, however, showed intraand inter-personal consistency and was independent of external light intensity and age. The observed independency from age confirming data from Fotiou [19], yet challenging data from other authors $[5,17,18]$ can yet be explained by the fact that the latency is primarily influenced by the speed of neuronal conduction through the optic and oculomotor nerve and speed of neuronal conduction is not age dependent [20].

The frequency of anisocoria (per our definition), its independence on gender or age and its persistence after light induced constriction are in line with earlier findings [21]; given the physiological Neurological Pupil index $(\mathrm{NPi}>3)$ in test persons exhibiting anisocor pupils, we could show that anisocoria may be physiological in about a quarter of the population. In that context, the latter underscores the need for a summarizing index of the PLR (as the NPi) in as much as its assessment based on the contemplation of a single value (size or CV) may lead to a misinterpretation on the PLR [22].

The PLR persisted significantly in conditions where subjects are exposed to direct light despite the fact that their pupil is already constricted. These reflexes generated a reduced RCA and, consequently, slower constriction and dilation velocities. This is due to the reduced mobility of the sphincter muscle when the iris is already pre-contracted [9]. The NPi, too, showed a decrement in the direct light exposure - although statistically significant, this decrement was very small.

One limitation of the present findings is the undetected contralateral PLR. We thus favour further pupillometric research including the contralateral pupil as its simultaneous examination could expand our understanding of the time course and extent of the bilateral neuronal and pupillary activity following retinal light stimulation in normal and pathological conditions (like in case of relative afferent pupillary defects). Understanding the precise quantitative nature of the PLR objectively (as opposed to the subjectivity of manual pupil assessments as it has been performed for decades) opens new scenarios in pathological conditions such as diabetes, traumatic brain injury or cardiac and other autonomic neuropathies [2, 23-25]. Thus, quantitative pupillometry represents a reliable tool to objectively evaluate the PLR and hence the autonomic nervous system.

\section{Conflict of Interest}

The authors declare that they have no competing interests.

\section{Acknowledgements}

No financial disclosures. 


\section{References}

[1] Larson MD, Behrends M. Portable infrared pupillometry: a review. Anesth Analg 2015; 120 (6): 1242-53.

[2] Chen JW, Vakil-Gilani K, Williamson KL, Cecil S. Infrared pupillometry, the Neurological Pupil index and unilateral pupillary dilation after traumatic brain injury: implications for treatment paradigms. Springerplus 2014; 3548.

[3] Du R, Meeker M, Bacchetti P, Larson MD, Holland MC, Manley GT. Evaluation of the portable infrared pupillometer. Neurosurgery 2005; 57 (1): 198-203; discussion 198-203.

[4] Olson DM, Stutzman S, Saju C, Wilson M, Zhao W, Aiyagari V. Interrater Reliability of Pupillary Assessments. Neurocrit Care 2016; 24 (2): 251-7.

[5] Taylor WR, Chen JW, Meltzer H, Gennarelli TA, Kelbch C, Knowlton S, Richardson J, Lutch MJ, Farin A, Hults KN, Marshall LF. Quantitative pupillometry, a new technology: normative data and preliminary observations in patients with acute head injury. Technical note. $J$ Neurosurg 2003; 98 (1): 205-13.

[6] Muppidi S, Adams-Huet B, Tajzoy E, Scribner M, Blazek P, Spaeth EB, Frohman E, Davis S, Vernino S. Dynamic pupillometry as an autonomic testing tool. Clin Auton Res 2013; 23 (6): 297-303.

[7] Bremner FD. Pupillometric evaluation of the dynamics of the pupillary response to a brief light stimulus in healthy subjects. Invest Ophthalmol Vis Sci 2012; 53 (11): 7343-7.

[8] Sharma S, Baskaran M, Rukmini AV, Nongpiur ME, Htoon H, Cheng CY, Perera SA, Gooley JJ, Aung T, Milea D. Factors influencing the pupillary light reflex in healthy individuals. Graefes Arch Clin Exp Ophthalmol 2016; 254 (7): 1353-9.

[9] Privitera CM, Stark LW. A binocular pupil model for simulation of relative afferent pupil defects and the swinging flashlight test. Biol Cybern 2006; 94 (3): 215-24.

[10] Chen JW, Gombart ZJ, Rogers S, Gardiner SK, Cecil S, Bullock RM. Pupillary reactivity as an early indicator of increased intracranial pressure: The introduction of the Neurological Pupil index. Surg Neurol Int 2011; 282.

[11] R Core Team. A language and environment for statistical computing. R Foundation for Statistical Computing, Vienna, Austria. 2018.

[12] Huber A, Kömpf D. Neuroophthalmology. Stuttgart: Georg Thieme Verlag, 1998.

[13] Boev AN, Fountas KN, Karampelas I, Boev C, Machinis TG, Feltes C, Okosun I, Dimopoulos V, Troup C. Quantitative pupillometry: normative data in healthy pediatric volunteers. $J$ Neurosurg 2005; 103 (6 Suppl): 496-500.

[14] Martinez-Ricarte F, Castro A, Poca MA, Sahuquillo J, Exposito L, Arribas M, Aparicio J. Infrared pupillometry. Basic principles and their application in the non-invasive monitoring of neurocritical patients. Neurologia 2013; 28 (1): 41-51.

[15] Rickmann A, Waizel M, Kazerounian S, Szurman P, Wilhelm H, Boden KT. Digital Pupillometry in Normal Subjects. Neuroophthalmology 2017; 41 (1): 12-18.

[16] Sachsenweger R. Neuroophthalmology. Leipzig: VEB Georg Thieme Verlag, 1977.

[17] Pfeifer MA, Weinberg CR, Cook D, Best JD, Reenan A, Halter JB. Differential changes of autonomic nervous system function with age in man. Am J Med 1983; 75 (2): 249-58.

[18] Tekin K, Sekeroglu MA, Kiziltoprak H, Doguizi S, Inanc M, Yilmazbas P. Static and dynamic pupillometry data of healthy individuals. Clin Exp Optom 2018.

[19] Fotiou DF, Brozou CG, Tsiptsios DJ, Fotiou A, Kabitsi A, Nakou M, Giantselidis C, Goula A. Effect of age on pupillary light reflex: evaluation of pupil mobility for clinical practice and research. Electromyogr Clin Neurophysiol 2007; 47 (1): $11-22$.

[20] Smith DO, Rosenheimer JL. Factors governing speed of action potential conduction and neuromuscular transmission in aged rats. Exp Neurol 1984; 83 (2): 358-66.

[21] Thurtell MJ, Tomsak RL, Daroff RB. Neuroophthalmology. Oxford - New York: Oxford University Press, 2012.

[22] Shoyombo I, Aiyagari V, Stutzman SE, Atem F, Hill M, Figueroa SA, Miller C, Howard A, Olson DM. Understanding the Relationship Between the Neurologic Pupil Index and Constriction Velocity Values. Sci Rep 2018; 8 (1): 6992.

[23] Lerner AG, Bernabe-Ortiz A, Ticse R, Hernandez A, Huaylinos Y, Pinto ME, Malaga G, Checkley W, Gilman RH, Miranda JJ, Group CCS. Type 2 diabetes and cardiac autonomic neuropathy screening using dynamic pupillometry. Diabet Med 2015; 32 (11): 1470-8.

[24] Jahns FP, Miroz JP, Messerer M, Daniel RT, Taccone FS, Eckert P, Oddo M. Quantitative pupillometry for the monitoring of intracranial hypertension in patients with severe traumatic brain injury. Crit Care 2019; 23 (1): 155.

[25] Oddo M, Sandroni C, Citerio G, Miroz JP, Horn J, Rundgren M, Cariou A, Payen JF, Storm C, Stammet P, Taccone FS. Quantitative versus standard pupillary light reflex for early prognostication in comatose cardiac arrest patients: an international prospective multicenter double-blinded study. Intensive Care Med 2018; 44 (12): 2102-2111. 